\author{
Jen-San Chen ${ }^{1}$ \\ Professor \\ e-mail: jschen@ntu.edu.tw \\ Yong-Zhi Lin \\ Graduate Student \\ Department of Mechanical Engineering, \\ National Taiwan University, \\ Taipei 10617, Taiwan
}

\title{
Snapping of a Planar Elastica With Fixed End Slopes
}

In this paper, we study the deformation and stability of a planar elastica. One end of the elastica is clamped and fixed in space. The other end of the elastica is also clamped, but the clamp itself is allowed to slide along a linear track with a slope different from that of the fixed clamp. The elastica deforms after it is subjected to an external pushing force on the moving clamp. It is observed that when the pushing force reaches a critical value, snapping may occur as the elastica jumps from one configuration to another remotely away from the original one. In the theoretical investigation, we calculate the static load-deflection curve for a specified slope difference between the fixed clamp and the moving clamp. To study the stability of the equilibrium configuration, we superpose the equilibrium configuration with a small perturbation and calculate the natural frequencies of the deformed elastica. An experimental setup is designed to measure the loaddeflection curve and the natural frequencies of the elastica. The measured load-deflection relation agrees with the theoretical prediction very well. On the other hand, the measured natural frequencies do not agree very well with the theoretical prediction, unless the mass of the moving clamp is taken into account. [DOI: 10.1115/1.2871207]

\section{Introduction}

Snap-through buckling in structures is a violent transition from one stable equilibrium position to another equilibrium position remotely away from the original one. For instance, a shallow arch with both ends fixed in space and under transverse loading may undergo snap-through buckling when certain conditions on the arch height and load are met; see the pioneering works of Timoshenko [1] and Bruce and Hoff [2]. Similar snap-through buckling phenomena can also be observed in the case of a shallow shell under lateral load; see the work of Budiansky and Roth [3]. Many more references on the snap-through phenomena in structures, such as shallow arches, shallow spherical caps, and cylindrical shells, can be found in the book by Simitses [4]. The term "snap through" is adopted because during buckling the structures snap from one side of the base plane "through" to the other side.

In this paper, we present a new snapping phenomenon observed in a clamped-clamped planar elastica, in which one clamp is fixed in space while the other is sliding along a linear track with a slope different from the one at the other end. Both theoretical and experimental results are presented. This structure may be used as a compliant mechanism in mechanical design [5,6]. In transmission of motion or force between two locations, compact space such as in miniature devices usually makes the conventional rigid body joint pairs impractical. In such a case, a flexible elastica capable of large deformation may be the only choice.

\section{Problem Formulation}

We consider a uniform beam with length $L$ and flexural rigidity $E I$. The two ends of the beam are denoted as $A$ and $B$, as shown in Fig. 1. An $x y$-coordinate system with origin attached to End $A$ is chosen to describe the geometry of the beam. The rotation angles of the beam at Ends $A$ and $B$ with respect to the $x$ axis are specified as $\theta_{A}=0$ and $\theta_{B}$, respectively. End $B$ is clamped and fixed in space. End $A$ is also clamped, but the clamp itself is allowed to slide on a straight line. In its initial configuration without any external pushing force in the longitudinal direction at End $A$, the

\footnotetext{
${ }^{1}$ Corresponding author.

Contributed by the Applied Mechanics Division for publication in the Journal of ApPlied Mechanics. Manuscript received July 30, 2007; final manuscript received November 8, 2007; published online May 20, 2008. Review conducted by Edmundo Corona.
}

neutral axis of the beam is a circular arc with radius $r$. The bending moment along the deformed beam is a constant in this initial configuration. It is noted that the beam may be termed an elastica, whose mechanics investigation was initiated by Euler and Lagrange over two centuries ago [7]. The relations between some geometric parameters are

$$
L=r \theta_{B}, \quad x_{B}=r \sin \theta_{B}, \quad y_{B}=r\left(1-\cos \theta_{B}\right)
$$

where $x_{B}$ and $y_{B}$ are the $x, y$ coordinates of Point $B$. The equations governing the motion of the elastica will be formulated first.

Figure 2 shows the free body diagram of an element $d s$ of the elastica after End $A$ is pushed in a distance $\delta_{A}$. Forces $F_{x}$ and $F_{y}$ are in the $x$ and $y$ directions, and $M$ is the bending moment. $\theta$ is the rotation angle of the tangent. First of all, from geometric relation we can write

$$
\begin{aligned}
& \frac{\partial x}{\partial s}=\cos \theta \\
& \frac{\partial y}{\partial s}=\sin \theta
\end{aligned}
$$

where $s$ is the length of the elastica measured from Point $A$. From the balance of moment and forces in the $x$ and $y$ directions, we can derive the following three equations:

$$
\begin{gathered}
\frac{\partial M}{\partial s}=F_{x} \sin \theta-F_{y} \cos \theta \\
\frac{\partial F_{x}}{\partial s}=\mu \frac{\partial^{2} x}{\partial t^{2}} \\
\frac{\partial F_{y}}{\partial s}=\mu \frac{\partial^{2} y}{\partial t^{2}}
\end{gathered}
$$

where $\mu$ is the mass per unit length of the elastica. $x(s, t)$ and $y(s, t)$ represent the deformed shape of the elastica. The momentcurvature equation from Euler-Bernoulli beam model is

$$
\frac{\partial \theta}{\partial s}=\frac{M}{E I}
$$

The six equations (2)-(7) govern the motion of the elastica. In the case when only static deformation is of interest, the terms involv- 


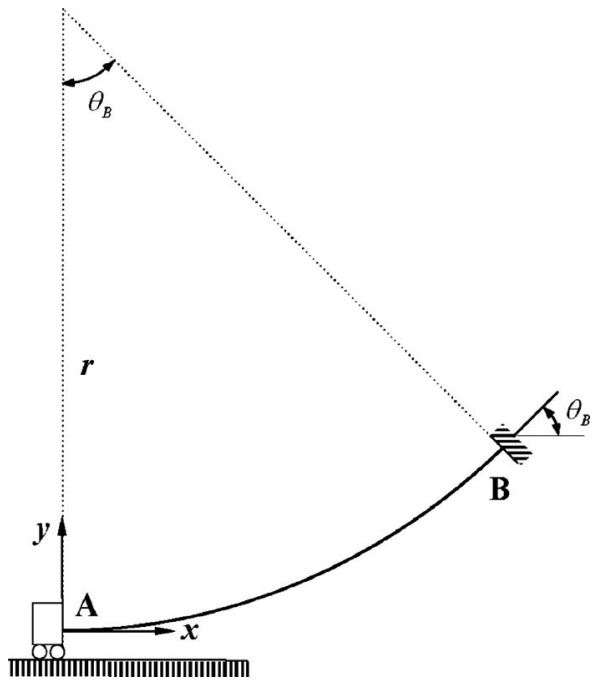

Fig. 1 A beam placed between the two clamps with specified directions. End $A$ of the beam is pushed in a distance along the horizontal direction, while End $B$ is fixed in space.

ing differentiation with respect to time in Eqs. (5) and (6) can be neglected.

\section{Static Deformation}

We first study the static deformation of the elastica after End $A$ is pushed in a distance $\delta_{A}$. There exist three different types of deformation configurations; they are the deformations with zero, one, and two inflection points. In this paper, we present the solution procedure for the deformation with two inflection points. The solution procedures for the other two simpler cases with zero and one inflection point are similar and can be found in the thesis of the second author [8].

Figure 3 shows an elastica with two inflection Points $C$ and $D$. End $A$ is under longitudinal force $P_{A}$ and is pushed in a distance $\delta_{A}$ in the horizontal direction. The shear force and bending moment at End $A$ are $Q_{A}$ and $M_{A}$, respectively. It is noted that in this static analysis we use the tangential component $P$ (longitudinal force) and the normal component $Q$ (shear force) on the cross section instead of using $F_{x}$ and $F_{y}$, as depicted in Fig. 2. $F_{x}$ and $F_{y}$ will be used in dynamic analysis later. $P$ and $Q$ can be related to $F_{x}$ and $F_{y}$ in the following relations:

$$
P=-F_{x} \cos \theta-F_{y} \sin \theta, \quad Q=-F_{x} \sin \theta+F_{y} \cos \theta
$$

The moment equation at any point $(x, y)$ of the elastica can be written as

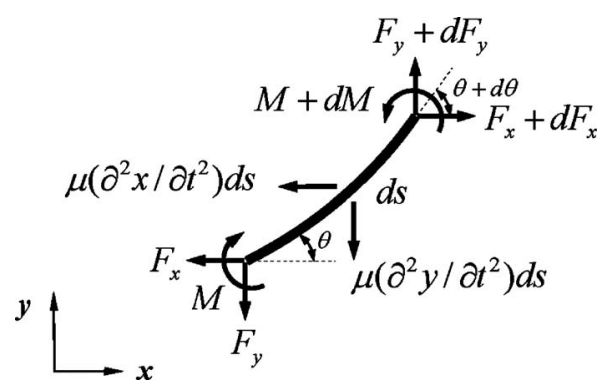

Fig. 2 The free body diagram of a small element $d s$

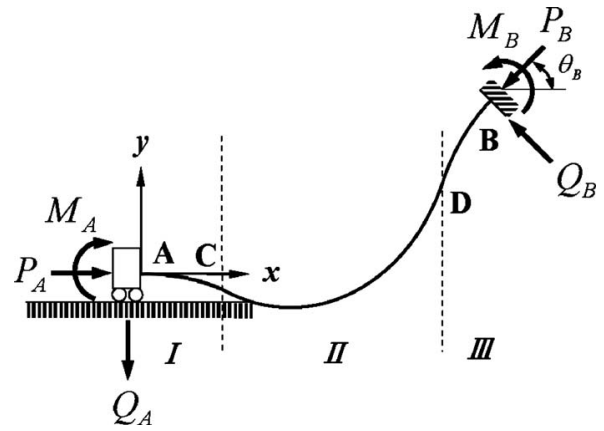

Fig. 3 Elastica deformation with two inflection points

$$
E I \frac{d \theta}{d s}=M_{A}-P_{A} y-Q_{A} x
$$

For convenience, we introduce the following dimensionless parameters (with asterisks):

$$
\begin{gathered}
\left(s^{*}, x^{*}, y^{*}, \delta_{A}^{*}\right)=\frac{\left(s, x, y, \delta_{A}\right)}{L}, \\
\left(P^{*}, Q^{*}, F_{x}^{*}, F_{y}^{*}\right)=\frac{L^{2}}{4 \pi^{2} E I}\left(P, Q, F_{x}, F_{y}\right)
\end{gathered}
$$

$M^{*}=\frac{L}{4 \pi^{2} E I} M, \quad t^{*}=\frac{1}{L^{2}} \sqrt{\frac{E I}{\mu}} t, \quad \omega^{*}=L^{2} \sqrt{\frac{\mu}{E I}} \omega, \quad m_{c}^{*}=\frac{1}{\mu L} m_{c}$

where $\omega$ is a circular frequency of the elastica. $m_{c}$ is the mass of the moving clamp, which will be used in dynamic analysis later. It is noted that $P^{*}=1$ corresponds to the Euler buckling load of a clamped-clamped straight beam. After substituting the above relations into Eq. (9) and dropping the asterisks thereafter for simplicity, Eq. (9) can be written in dimensionless form as

$$
\frac{d \theta}{d s}=4 \pi^{2}\left(M_{A}-P_{A} y-Q_{A} x\right)
$$

Similarly, Eqs. (5)-(7) can be rewritten in the following forms:

$$
\begin{gathered}
\frac{\partial F_{x}}{\partial s}=\frac{1}{4 \pi^{2}} \frac{\partial^{2} x}{\partial t^{2}} \\
\frac{\partial F_{y}}{\partial s}=\frac{1}{4 \pi^{2}} \frac{\partial^{2} y}{\partial t^{2}} \\
\frac{\partial \theta}{\partial s}=4 \pi^{2} M
\end{gathered}
$$

It is noted that the dimensionless versions of Eqs. (2)-(4) remain the same as the dimensional versions. By differentiating Eq. (10) with respect to $s$ once, and using the relations (2) and (3), we obtain

$$
\frac{d^{2} \theta}{d s^{2}}=-4 \pi^{2}\left(P_{A} \sin \theta+Q_{A} \cos \theta\right)
$$

We multiply Eq. (11) by $d \theta$ and integrate to obtain

$$
\left(\frac{d \theta}{d s}\right)^{2}=8 \pi^{2}\left(P_{A} \cos \theta-Q_{A} \sin \theta+K\right)
$$

where $K$ is an integration constant. The sign of the curvature $d \theta / d s$ can be positive or negative, depending on the deformation pattern. The slopes of the elastica at Points $C$ and $D$ are denoted as $\alpha$ and $\beta$, respectively. We divide the domain of the elastica into three segments as separated by Points $C$ and $D$. In Segments I and 
III, the curvature of the elastica is negative, while in Segment II, the curvature is positive.

Segment I must satisfy the condition $d \theta / d s=0$ when $\theta=\alpha$. Therefore, for Segment I the integration constant $K$ can be found as

$$
K=Q_{A} \sin \alpha-P_{A} \cos \alpha
$$

As a consequence, Eq. (12) can be written as

$$
\left(\frac{d \theta}{d s}\right)_{\mathrm{I}}^{2}=8 \pi^{2}\left[P_{A}(\cos \theta-\cos \alpha)-Q_{A}(\sin \theta-\sin \alpha)\right]
$$

After taking square root, we have the curvature in Segment I,

$$
\left(\frac{d \theta}{d s}\right)_{\mathrm{I}}=-2 \sqrt{2} \pi\left[P_{A}(\cos \theta-\cos \alpha)-Q_{A}(\sin \theta-\sin \alpha)\right]^{1 / 2}
$$

From Eq. (15), the length of the elastica in Segment I can be calculated as

$$
l_{\mathrm{I}}=-\int_{0}^{\alpha} \frac{d \theta}{2 \sqrt{2} \pi \sqrt{P_{A}(\cos \theta-\cos \alpha)-Q_{A}(\sin \theta-\sin \alpha)}}
$$

The $x$ and $y$ coordinates of Point $C$ relative to $A$ can be calculated as

$$
\begin{aligned}
& x_{C / A}=-\int_{0}^{\alpha} \frac{\cos \theta d \theta}{2 \sqrt{2} \pi \sqrt{P_{A}(\cos \theta-\cos \alpha)-Q_{A}(\sin \theta-\sin \alpha)}} \\
& y_{C / A}=-\int_{0}^{\alpha} \frac{\sin \theta d \theta}{2 \sqrt{2} \pi \sqrt{P_{A}(\cos \theta-\cos \alpha)-Q_{A}(\sin \theta-\sin \alpha)}}
\end{aligned}
$$

The situation in Segment III is similar to the one in Segment I. The equation for curvature $(d \theta / d s)_{\mathrm{III}}$ is the same as in Eq. (15) except that $\alpha$ is replaced by $\beta$. The formulas for $l_{\mathrm{III}}, x_{B / D}$, and $y_{B / D}$ are the same as in Eqs. (16)-(18), except that $\alpha$ is replaced by $\beta$ in all the integrands, and the integration limits are changed to from $\beta$ to $\theta_{B}$.

Segment II must satisfy the condition $d \theta / d s=0$ when $\theta=\alpha$ and $\beta$. Therefore, the integration constant $K$ can be found as

$$
K=Q_{A} \sin \alpha-P_{A} \cos \alpha=Q_{A} \sin \beta-P_{A} \cos \beta
$$

As a consequence, $Q_{A}$ and $P_{A}$ must satisfy the relation

$$
Q_{A}=P_{A} \frac{\cos \alpha-\cos \beta}{\sin \alpha-\sin \beta}
$$

The formulas for $l_{\mathrm{II}}, x_{D / C}$, and $y_{D / C}$ are the same as in Eqs. (16)-(18), except that $\alpha$ is replaced by $\beta$, and the integration limits are changed to from $\beta$ to $\alpha$.

We assume that the elastica is inextensible. As a consequence, we have the relation

$$
l_{\mathrm{I}}+l_{\mathrm{II}}+l_{\mathrm{III}}=1
$$

The relative position between Ends $B$ and $A$ in the $y$ direction is fixed; therefore, we have

$$
y_{B / D}+y_{D / C}+y_{C / A}=y_{B / A}=\frac{1-\cos \theta_{B}}{\theta_{B}}
$$

By specifying $P_{A}$ and $\theta_{B}$, and using Eqs. (20)-(22), we can solve for the three unknowns $Q_{A}, \alpha$, and $\beta$. The displacement $\delta_{A}$ of End $A$ in the horizontal direction can then be calculated from

$$
\delta_{A}=\frac{\sin \theta_{B}}{\theta_{B}}-\left(x_{B / D}+x_{D / C}+x_{C / A}\right)
$$

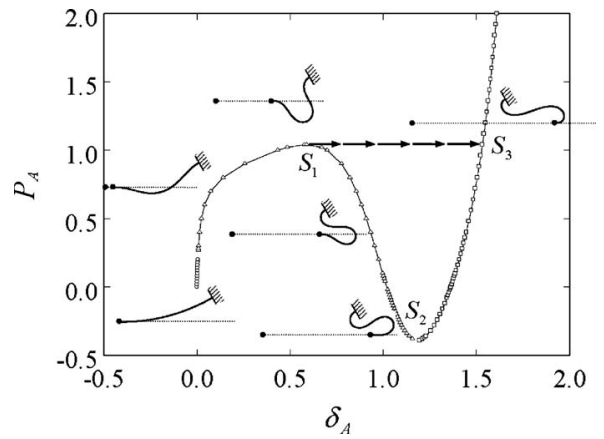

Fig. 4 Load-deflection curve for $\boldsymbol{\theta}_{B}=30$ deg. The symbols $\bigcirc$, $\square$, and $\triangle$ represent deformations without, with one, and with two inflection points, respectively.

\section{Load-Deflection Curve When $\boldsymbol{\theta}_{B}=\mathbf{3 0} \mathrm{deg}$}

Figure 4 shows the relation between the longitudinal force $P_{A}$ and end movement $\delta_{A}$ for the case when $\theta_{B}=30 \mathrm{deg}$. This curve is also called the load-deflection curve. The symbols $\bigcirc, \square$, and $\triangle$ represent deformations without, with one, and with two inflection points, respectively. The deformed configurations of the elastica at various stages are also depicted. The two black dots on the dashed horizontal line represent the starting point and the stop point of End $A$. The elastica is originally in the circular arc shape when $P_{A}$ is zero, which has no inflection point. The elastica deformation experiences a transition from no inflection point to two inflection points when $\left(P_{A}, \delta_{A}\right)=(0.25,0.006)$, and experiences a transition from two inflection points to one inflection point when $\left(P_{A}, \delta_{A}\right)$ $=(-0.39,1.19)$. The load-deflection curve approaches a local maximum $S_{1}$ at $\left(P_{A}, \delta_{A}\right)=(1.04,0.58)$ and hits a local minimum $S_{2}$ at $\left(P_{A}, \delta_{A}\right)=(-0.4,1.19)$. It is noted that a negative $P_{A}$ means that a pulling force is required to maintain equilibrium of the elastica. The load-deflection curve between $S_{1}$ and $S_{2}$ has negative slope.

It is noted that the above static analysis does not say anything about the stability of the equilibrium positions. Previous experience with shallow arches and shallow shells suggests that the equilibrium positions corresponding to the load-deflection curve with negative slope are unstable [9]. Therefore, the equilibrium positions corresponding to the deflection curve with negative slope cannot be realized in the laboratory. As a consequence, if we follow the load-deflection curve by increasing the external pushing force $P_{A}$ to slightly beyond the local maximum $S_{1}$, the elastica will snap, as indicated by the arrows, to an equilibrium position corresponding to a Point $S_{3}$ on the load-deflection curve. This conjecture will be examined by a dynamic analysis in the next section.

\section{Natural Frequencies and Stability Analysis}

In this section, we study the natural frequencies and stability of the elastica when it is pushed in by a distance $\delta_{A}$. We first solve for the static deformation of the elastica as described in Secs. 3 and 4 , and denote the static solution as $x_{e}(s), y_{e}(s), \theta_{e}(s), M_{e}(s)$, $F_{x e}(s)$, and $F_{y e}(s)$. We superpose the static solution with small perturbation, for instance,

$$
x(s, t)=x_{e}(s)+x_{d}(s) \sin \omega t
$$

where $\omega$ is a natural frequency. The other variables $y(s, t), \theta(s, t)$, $M(s, t), F_{x}(s, t)$, and $F_{y}(s, t)$ are treated in the same manner.

By substituting the above perturbed variables into Eqs. (2)-(4) and $\left(5^{\prime}\right)-\left(7^{\prime}\right)$ and ignoring the higher order terms, we arrive at the following linear equations for the six unknowns $x_{d}(s), y_{d}(s)$, $\theta_{d}(s), M_{d}(s), F_{x d}(s)$, and $F_{y d}(s)$ : 


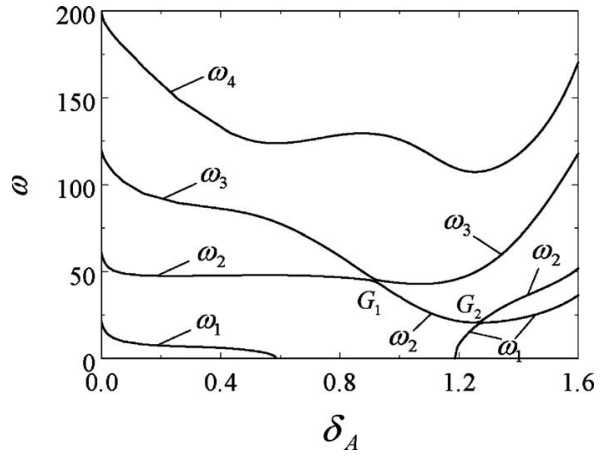

Fig. 5 The first four natural frequencies as functions of $\delta_{A}$ for $\theta_{B}=30 \mathrm{deg}$

$$
\begin{gathered}
\frac{\partial x_{d}}{\partial s}=-\theta_{d} \sin \theta_{e}, \quad \frac{\partial y_{d}}{\partial s}=\theta_{d} \cos \theta_{e}, \quad \frac{\partial \theta_{d}}{\partial s}=4 \pi^{2} M_{d} \\
\frac{\partial M_{d}}{\partial s}=\left(F_{x e} \theta_{d}-F_{y d}\right) \cos \theta_{e}+\left(F_{x d}+F_{y e} \theta_{d}\right) \sin \theta_{e} \\
\frac{\partial F_{x d}}{\partial s}=-\frac{1}{4 \pi^{2}} \omega^{2} x_{d}, \quad \frac{\partial F_{y d}}{\partial s}=-\frac{1}{4 \pi^{2}} \omega^{2} y_{d}
\end{gathered}
$$

The boundary conditions at $s=1$ are

$$
x_{d}(1)=y_{d}(1)=\theta_{d}(1)=0
$$

It is noted that while End $A$ is subjected to a prescribed pushing force $P_{A}$, it is allowed to slide freely when the elastica vibrates. As a consequence, the boundary conditions at $s=0$ are

$$
F_{x d}(0)=y_{d}(0)=\theta_{d}(0)=0
$$

In other words, this is a load-control procedure. It is noted that $\omega$ in linear equations (25)-(27) only appears in the form of $\omega^{2}$. Therefore, if the characteristic value $\omega^{2}$ is positive, it means that the corresponding mode is stable with natural frequency $\omega$. On the other hand, if $\omega^{2}$ is negative, it means that the equilibrium position is unstable. The Newton-Raphson method is used to solve for the characteristic values $\omega^{2}$. The details of the numerical procedure can be found in Ref. [8].

Figure 5 shows the first four natural frequencies as functions of the end movement $\delta_{A}$ for the case when $\theta_{B}=30 \mathrm{deg}$. Two important features should be noted here. First of all, we notice that the lowest natural frequency $\omega_{1}$ is 21 when $\delta_{A}=0$. As $\delta_{A}$ increases to $0.58, \omega_{1}$ decreases to zero. Between $\delta_{A}=0.58$ and $1.19, \omega_{1}$ is purely imaginary, and the elastica is unstable. This range of $\delta_{A}$ corresponds to the load-deflection curve between $S_{1}$ and $S_{2}$ in Fig. 4. This analysis validates the conjecture we made in Sec. 4 that the load-deflection curve between $S_{1}$ and $S_{2}$ in Fig. 4 represents the unstable equilibrium positions.

The second feature worthy of a closer look is that the frequency loci in Fig. 5 appear to be crossing each other at locations $G_{1}$ $\left(\delta_{A}=0.92\right)$ and $G_{2}\left(\delta_{A}=1.27\right)$. However, if we magnify the frequency loci near the neighborhood of these two locations, the frequency loci are actually veering away from each other. Figure 6 shows the magnification of the frequency loci near $G_{1}$. In the same figure, we also show the mode shapes corresponding to the four natural frequencies in the neighborhood. The dotted lines represent the equilibrium shapes. The solid lines represent the mode shapes. The intersections of the dotted lines and the solid lines are the nodal points of the mode shapes, as noted by the open circles. It is noted that the two mode shapes on the top possess the three nodal points. The left mode shape on the bottom possesses two nodal points, while the one on the right possesses only one

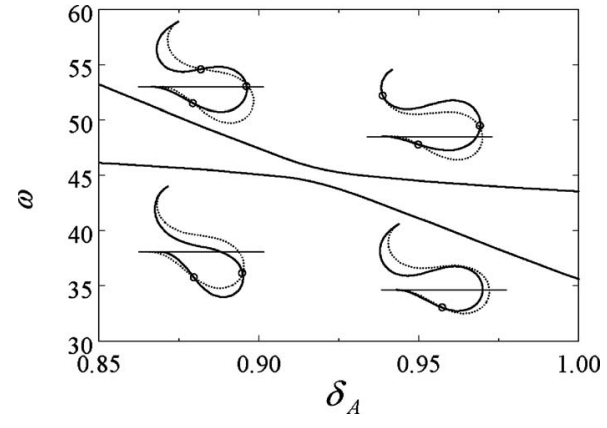

Fig. 6 Magnification of the frequency loci near location $G_{1}$ in Fig. 5. The mode shapes corresponding to the natural frequencies are also shown.

nodal point. It is interesting to note that a similar frequency loci veering phenomenon occurs in other totally different structures, such as in rotating flexible disks [10].

\section{Experiments}

Figure 7 shows a schematic diagram of the experimental setup we use to examine the theoretical predictions. The elastica is made of a stainless steel strip (AISI type 420) with Young's modulus $200 \mathrm{GPa}$ and a mass density of $7800 \mathrm{~kg} / \mathrm{m}^{3}$. The length $L$ of the strip is $60 \mathrm{~cm}$ and the cross section is $60 \times 0.3 \mathrm{~mm}^{2}$. One end of the strip is fixed in an aluminum clamp, while the other aluminum clamp on the other end is allowed to slide on a guide rail. The angle between the fixed clamp and the sliding clamp is adjustable. For the time being, we set the angle to be $30 \mathrm{deg}$. The sliding clamp is attached by a cotton string. The string passes through a pulley with a bucket attached to the other end. In the bucket, we put in small steel screws as dead load. Each small screw weighs $11.2 \mathrm{~g}$. The displacement of the sliding clamp can be recorded by using a ruler. In order to measure the natural frequencies of the elastica under fixed load, we point a photonic probe (MTI 2000) normal to one point of the elastica. After hitting the elastica manually, we can obtain the natural frequencies of the system.

The cross marks $(\times)$ in Fig. 8 represent the measured loaddeflection relation. For convenient reference, we present the measured results with both dimensionless parameters (left and bottom sides) and the physical ones (right and top sides). The same labeling style is adopted in Fig. 9 as well. The solid line represents the theoretical prediction, as shown in Fig. 4. We found that the measured deflections agree with the theoretical predictions reasonably well.

The natural frequencies are obtained from a power spectrum measurement by hitting the elastica at about one-third of the length from the moving end (End $A$ ) and measuring the displacement of the elastica at a location close to the fixed end (End $B$ ). Figure 9 shows the power spectrum when the elastica is free from

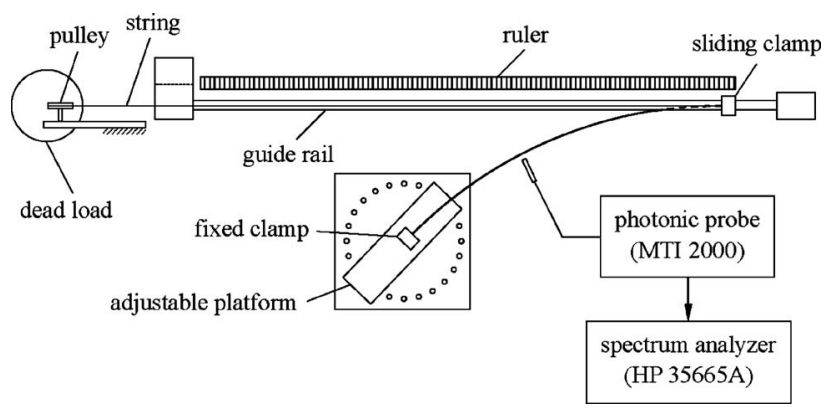

Fig. 7 Schematic diagram of the experimental setup 
(m)

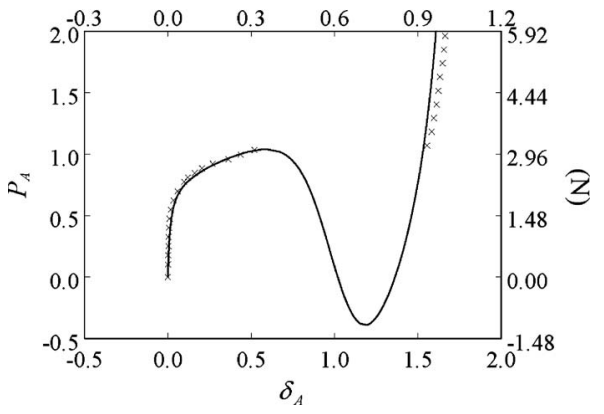

Fig. 8 Experimental measurement of load-deflection curve

external pushing force. Four natural frequencies can be observed within the range of $50 \mathrm{~Hz}$. The lowest natural frequency is $3.4 \mathrm{~Hz}$. As $\delta_{A}$ increases, this lowest natural frequency approaches zero quickly and is difficult to measure. In Fig. 10, we record the variation of these four natural frequencies with cross marks $(X)$ as $\delta_{A}$ changes due to the external load. To compare these measured natural frequencies with the theoretical predictions, we replot the theoretical natural frequency loci from Fig. 5 as dashed lines. It is found that the measured natural frequencies do not agree with the

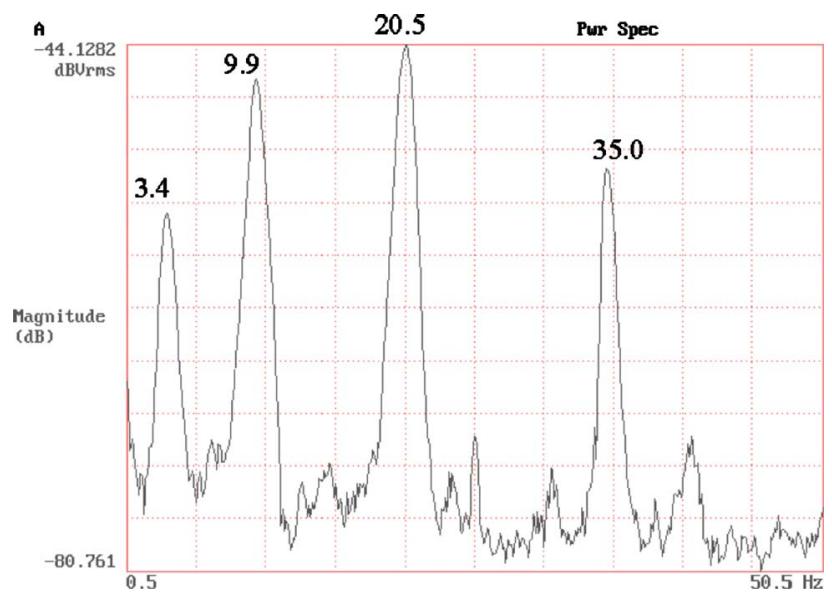

Fig. 9 Power spectrum when the elastica is free from external pushing force

(m)

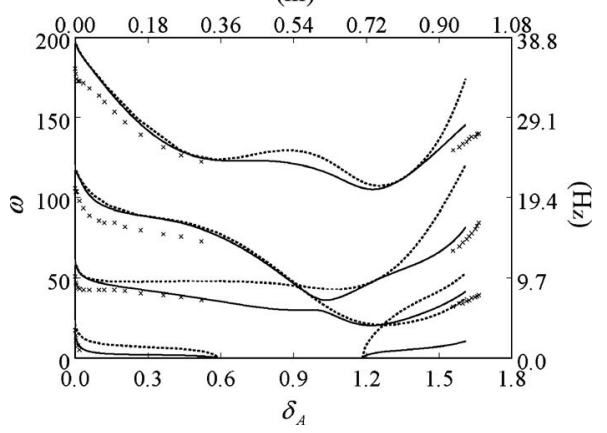

Fig. 10 The measured natural frequencies are recorded with cross marks. The dashed and solid curves are the theoretical predictions neglecting and including the clamp mass, respectively.

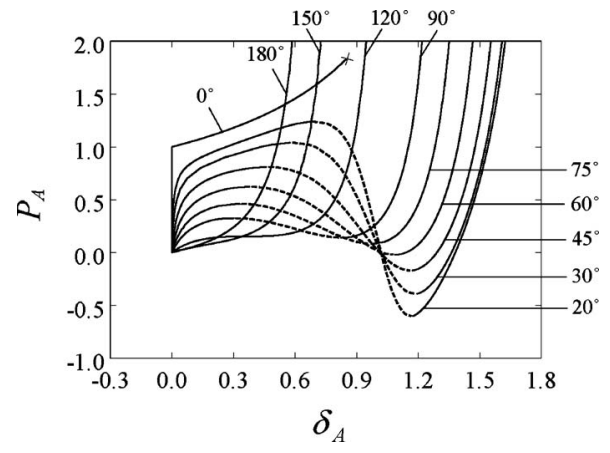

Fig. 11 Load-deflection curves for various values of $\theta_{B}$

theoretical curves from Fig. 5 very well. To account for this discrepancy, we have to take into account the mass of the moving clamp.

\section{Effect of Clamp Mass on the Natural Frequencies}

In Sec. 5, we calculate the natural frequencies of the elastica by ignoring the mass of the sliding clamp. In the experiment, the mass of the sliding clamp is $483 \mathrm{~g}$. This mass is quite significant compared to the mass of the elastica, $84 \mathrm{~g}$. In order to take into account the mass of the clamp, the boundary condition (with dimension) at the moving end must be modified as

$$
F_{x}(0, t)=m_{c} \frac{\partial^{2} x(0, t)}{\partial t^{2}}-P_{A}
$$

where $P_{A}=-F_{x e}(0) . m_{c}$ is the mass of the moving clamp. After following the same linearization procedure as in Sec. 5, the dimensionless boundary condition on $F_{x d}(0)$ in Eq. (29) can be replaced by

$$
F_{x d}(0)=-\frac{1}{4 \pi^{2}} m_{c} \omega^{2} x_{d}(0)
$$

After this modification, the predicted natural frequencies are plotted as the solid lines in Fig. 10. Generally speaking, the clamp mass lowers the natural frequencies. Besides, the clamp mass does not affect the range in which $\omega_{1}$ becomes imaginary. It is observed that the agreement between the measured natural frequencies and the theoretical prediction is improved by taking into account the clamp mass. It is noted that after snapping, we are unable to measure the lowest natural frequency because the friction force is too large due to the large shear force from the clamp. This sticking phenomenon from the friction force prevents the free movement of the moving clamp, which is essential to the fundamental mode. In this paper, we do not consider friction in our formulation.

\section{Effects of $\boldsymbol{\theta}_{B}$}

Having experimentally confirmed the theoretical predictions, we are ready to investigate the effect of $\theta_{B}$ on the load-deflection relations. Figure 11 shows the theoretical load-deflection curves for various $\theta_{B}$. In this figure, we identify the unstable equilibrium positions with dashed lines. The load-deflection curve for $\theta_{B}$ $=0 \mathrm{deg}$ is a special case and deserves more attention. For this case, End $A$ will not move until $P_{A}$ reaches the critical load $P_{A}$ $=1$, at which the natural frequency $\omega_{1}$ is zero. The first four natural frequencies as functions of $\delta_{A}$ are shown in Fig. 12. It is noted that this elastica becomes unsable when $\delta_{A}$ reaches 1 . It means that Ends $A$ and $B$ coincide. However, it is impossible to produce this equilibrium position in the laboratory without dismantling Clamp A and reversing its direction [11]. Furthermore, before $\delta_{A}$ reaches 1 the elastica will contact itself when $\delta_{A}=0.85$. This selfcontact phenomenon prevents Clamp A from moving beyond $\delta_{A}$ $=0.85[11,12]$. The deformation configurations corresponding to 


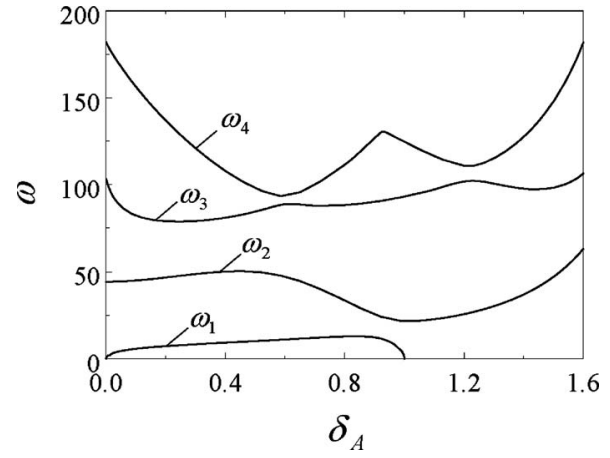

Fig. 12 The first four natural frequencies as functions of $\delta_{A}$ for $\theta_{B}=0 \mathrm{deg}$

$\delta_{A}=0.85$ and 1 are depicted in Figs. 13(a) and 13(b). The loaddeflection curve for $\theta_{B}=0$ deg in Fig. 11 is terminated with a cross mark when self-contact occurs. To avoid self-contact, $\theta_{B}$ must be greater than $18 \mathrm{deg}$. It is noted that for $\theta_{B}=120 \mathrm{deg}, 150 \mathrm{deg}$, and 180 deg no snapping will occur.

For the load-deflection curves in Fig. 11 with local maximum and local minimum, we denote the external force $P_{A}$ corresponding to the local maximum as $P_{A(\mathrm{cr})}^{+}$and the local minimum as $P_{A(\mathrm{cr})}^{-}$. If Clamp $\mathrm{A}$ is pushed in from the original position, the elastica will snap when $P_{A}$ reaches $P_{A(\mathrm{cr})}^{+}$. On the other hand, if we trace the load-deflection curve in the opposite direction by reducing $P_{A}$, then a reverse snapping may occur when $P_{A}$ reaches $P_{A(\mathrm{cr})}^{-}$. Figure 14 shows these two critical loads as functions of $\theta_{B}$. It is noted that snapping can occur only when $\theta_{B}$ is in the range from $18 \mathrm{deg}$ to $119 \mathrm{deg}$. The gap between $P_{A(\mathrm{cr})}^{+}$and $P_{A(\mathrm{cr})}^{-}$decreases as $\theta_{B}$ increases.

\section{Conclusions}

In this paper, we study, both theoretically and experimentally, the deformation and stability of a clamped-clamped planar elastica, with one clamp fixed in space and the other allowed to slide along a straight line when it is under a longitudinal pushing force. Several conclusions can be summarized as follows.

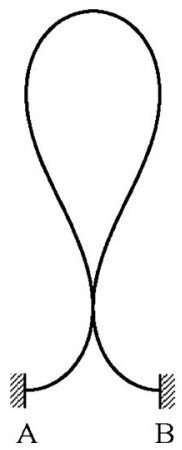

(a)

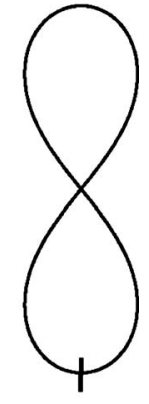

(b)
Fig. 13 (a) Self-contact occurs when $\delta_{A}=0.85$. (b) Clamp reverse is required for this deformation configuration when $\delta_{A}$ $=1$.

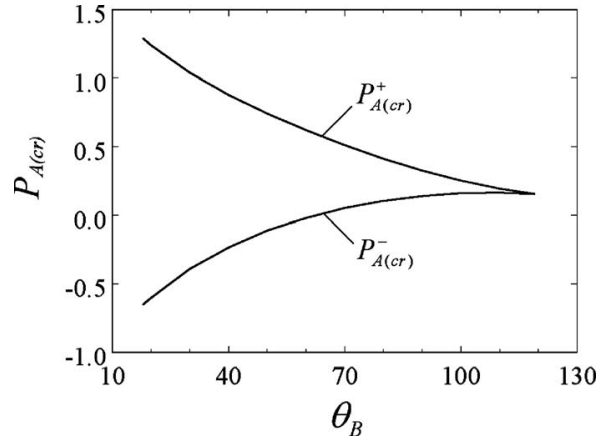

Fig. 14 Critical loads $P_{A(\mathrm{cr})}^{+}$and $P_{A(\mathrm{cr})}^{-}$as functions of $\theta_{B}$

(1) The deformation of the elastica can have zero, one, and two inflection points, depending on the slope difference between the two ends and the magnitude of the external longitudinal force.

(2) When the pushing force reaches a critical value, snapping may occur as the elastica jumps from one configuration to another remotely away from the original one. The equilibrium configuration corresponding to a negative slope in the load-deflection curve is unstable.

(3) Natural frequency loci veering phenomena are observed when the natural frequencies of the elastica are plotted as functions of the sliding clamp movement.

(4) The measured static load-deflection relation agrees with the theoretical prediction very well. On the other hand, the measured natural frequencies do not agree very well with the theoretical prediction, unless the mass of the moving clamp is taken into account.

\section{References}

[1] Timoshenko, S. P., 1935, "Buckling of Flat Curved Bars and Slightly Curved Plates," ASME J. Appl. Mech., 2, pp. 17-20.

[2] Hoff, N. J., and Bruce, V. G., 1954, "Dynamic Analysis of the Buckling of Laterally Loaded Flat Arches," J. Math. Phys. (Cambridge, Mass.), 32, pp. 276-288.

[3] Budiansky, B., and Roth, R. S., 1962, "Axisymmetric Dynamic Buckling of Clamped Shallow Spherical Caps," Collected Papers on Instability of Shells Structures, NASA Paper No. TND-1510, pp. 597-606.

[4] Simitses, G. J., 1990, Dynamic Stability of Suddenly Loaded Structures, Springer-Verlag, New York.

[5] Kimball, C., and Tsai, L.-W., 2002, "Modeling of Flexural Beams Subjected to Arbitrary End Loads," ASME J. Mech. Des., 124, pp. 223-235.

[6] Venanzi, S., Giesen, P., and Parenti-Castelli, V., 2005, "A Novel Technique for Position Analysis of Planar Compliant Mechanisms," Mech. Mach. Theory, 40, pp. 1224-1239.

[7] Love, A. E., 1944, A Treatise on the Mathematical Theory of Elasticity, Dover, New York.

[8] Lin, Y.-Z., 2007, Longitudinal Force Transmission and Vibration of a Planar Elastica, Master thesis, Department of Mechanical Engineering, National Taiwan University.

[9] Simitses, G. J., 1976, An Introduction to the Elastic Stability of Structures, Prentice-Hall, Englewood Cliffs, NJ.

[10] Chen, J.-S., and Bogy, D. B., 1992, "Mathematical Structure of Modal Interactions in a Spinning Disk-Stationary Load System," ASME J. Appl. Mech. 59, pp. 390-397.

[11] Van der Heijden, G. H. M., Neukirch, S., Goss, V. G. A., and Thompson, J. M. T., 2003, "Instability and Self-Contact Phenomena in the Writhing of Clamped Rods," Int. J. Mech. Sci., 45, pp. 161-196.

[12] Plaut, R. H., Taylor, R. P., and Dillard, D. A., 2004, "Postbuckling and Vibration of a Flexible Strip Clamped at its Ends to a Hinged Substrate," Int. J. Solids Struct., 41, pp. 859-870. 